\title{
Correlation Similarity Measure based Document Clustering with Directed Ridge Regression
}

\author{
R. Nagaraj ${ }^{1 *}$ and V. Thiagarasu ${ }^{2}$ \\ 'Karpagam University, Coimbatore, India; nagukasc@gmail.com \\ ²Computer Science, Gobi Arts \& Science College, Gobichettipalayam, India; profdravt@gmail.com
}

\begin{abstract}
Correlation Preserving Indexing (CPI) can discover the intrinsic structures implanted in high-dimensional document space. To predict the result of one variable based on another variable is not suitable for all the situations since two variable prediction problems takes places. In this paper, Directed Ridge Regression is introduced to predict two or more variables which are highly correlated in high dimensional document space. Directed Ridge Regression is a statistical technique to estimate the relationship among the variables based on the Eigen values to find the similarity between the documents. The directed ridge estimator alters the diagonal elements of the Eigen values. The objective of the Directed Ridge Regression is to achieve efficient document clustering in similarity measure. Experimental results shows that compared to Correlation Preserving Indexing, the Directed Ridge Regression achieves efficient document clustering.
\end{abstract}

Keywords: Correlation Similarity Measure, Directed Ridge Regression, Document Clustering, Latent Semantic Indexing

\section{Introduction}

Document clustering is nothing but grouping the related documents into clustering. The document clustering is a considerable process in data mining and it is a general method which is used in extracting the topic, automatic document association and information retrieval. In document clustering, the Euclidean distance measure is most comprehensively utilized. Spectral clustering approaches are used for low computation cost where the documents are estimated into a low-dimensional semantic space and for identifying the document clusters the conventional clustering method is used. The latent semantic indexing is one of the proficient techniques in spectral clustering. The main intent of this method is to find the best subspace approximation to the inventive document space by diminishing the global reconstruction error. The Locality preserving indexing (LPI) technique is a spectral clustering approach which is based on graph separation theory ${ }^{1}$. BY using a weighted function, this method focuses on detaining the similarity structure of the documents ${ }^{1}$. It does not correct the drawback of Euclidean distance.
The correlation preserving indexing is a method which is suggested to deal with the difficulty of similarities between the documents. This Correlation preserving Indexing method primarily considers the manifold structure which is entrenched in the similarities between the documents. The intent of the Correlation preserving Indexing is to achieve a most sympathetic semantic subspace by concomitantly growing the associations between the documents in the local patches and to reduce the associations between the documents outside these patches. This method is similarity measure which is focused on identifying the intrinsic formation between close by documents. CPI can proficiently recognize the intrinsic semantic structure of the high-dimensional document space.

In the correlation preserving indexing there is a two variable problem. In the correlation method based on one variable one result can be predicted based on another variable. This becomes not suitable for every variable, and hence the efficient clustering cannot be achieved in this method. To address this trouble Directed Ridge Regression (DRR) is applied in order to bring the relationships

${ }^{*}$ Author for correspondence 
among the variables. It identifies the similarity between the documents by evaluation of relationship among the variables. In the regression analysis specifically Directed Ridge Regression (DRR) is used in which effective document clustering is accomplished.

\section{Literature Review}

A document separation method was proposed in order to accomplish the high correctness and capability for computation of the number of clusters in the set of documents ${ }^{2}$. A clustering is achieved by characterizing the document by a term-frequency vector with its magnitude to represent the number of times a specific word occurred in the document. A more affluent feature set is used to disseminate each and every document for precisely clustering the documents. The Gaussian Mixture Model together with the Expectation-Maximization approach is used to cluster more number of documents.

A new technique is called Locality preserving Indexing is utilized for document clustering ${ }^{3}$. The locality Preserving Indexing (LPI) is a method in which the documents can be anticipated into a lower-dimensional semantic space. The documents associated to the identical semantics are close up to each other. This method investigates to identify both the numerical and discriminating structures of the document space. The main intent of the Locality Preserving Indexing is to discover the global Euclidean structure. It can have more discerning power. The derivation of the LPI is accomplished by determining the most favorable linear estimation to the Eigen functions of the Laplace Beltrami operator on the document. The second order derivatives of the functions on the manifolds are utilized by the Laplace Beltrami operator. It estimates the softness of the functions. Resulting to that, it can determine the nonlinear manifold configuration to some extent.

Distributional Clustering was clarified for the document classification. Data clustering is a complicated process in information processing and pattern recognition $^{4}$. The dispute is both theoretical and computational. Impulsively, to cluster a dataset, the intention is to partition it into subsets such that points in the same subset are more "related" to each other than to points in other subsets. The usual clustering algorithms depend on choosing a similarity measure between data points and a "correct" clustering result can be dependent on an appropriate choice of a similarity measure. Based on the allocation of the class labels related with each word, this method clusters words into groups. The words are into groups predominantly for the document classification. In the document organization the focus point is class label. By allocating the class labels related with the words, measure word similarity. Distributional Clustering will cautiously cluster words that are investigative of more than one class.

For the correlation similarity determination a discriminant learning process is utilized ${ }^{5}$. This is one of the methods that explore for a global linear transformation to utilize the correlation of samples from the same class and diminish the correlation of samples from different classes in the transformed space. This method merges the similarity measure learning with the supervised discriminant learning in a very simple way to determine the nonlinear information instead of only linear information. In this method based on the description of within-class correlation and between-class correlation, the best alteration can be required to extend the dissimilarity between them, which is in conformance with good classification performance practically. The difference can be virtually calculated as a standard for the classification performance.

Semi-supervised learning that is based on a Gaussian random field model $^{6}$. In a weighted graph the vertices represented the labeled and unlabeled data, with edge weights encoding the similarity between instances. After that the learning problem is formulated in terms of Gaussian random field on this graph, where the mean of the field is differentiate in terms of harmonic functions, and is proficiently attained using matrix methods or belief propagation. In this method, the solution is exclusively based on the structure of the data manifold, which is consequent from data features. In practice, however, this derived manifold structure may be inadequate for precise classification. The fully probabilistic framework is intimately related to Gaussian process classification, and this connection proposes principled ways of incorporating class priors and learning hyper parameters.

The metric learning algorithm for text documents was introduced ${ }^{7}$. Based on the exploiting the inverse volume of a given data set of points, this method selecting a metric from a parametric family. The captured metric is local, thus detaining local variations within the space and is characterize on the entire embedding space. A group of metric candidates is represented as a parametric family of transformations. Based on the some performance 
criteria, the obtained metric is chosen. By examining the application of the metric learning techniques in the context of classification of text documents and images, the experimental results for text classification was provided.

For a given set of documents, a document clustering method was suggested based on the non-negative factorization of the term document matrix ${ }^{8}$. This method is used to compute the latent semantic space whereas every axis confines the base topic of particular documents. Also each and every document is disseminated as an additive grouping of the base topics. In every document the cluster membership can be effortlessly determined by classifying the base topic with which the document has the main projection value. This method is based on the singular vector decomposition and the associated spectral clustering methods in that the latent semantic space derived by NMF does not need to be orthogonal, and that each document is definite to take only non-negative values in all the latent semantic directions.

Spatial data mining is the proficient and effectual for the clustering approaches'. This method is used to recognize the relations and distinctiveness that may endure completely in spatial databases. The main intent of the spatial data mining is to mechanize such a knowledge detection process. The major role is to remove the interesting spatial patterns and features, detaining essential associations between spatial and non-spatial data, presenting data reliability succinctly and at higher hypothetical levels, helping to rearrange spatial databases to comprise data semantics and also attain better performance. However the quality of the results formed by both algorithms relies quite critically on the appropriateness of the hierarchy to the given data.

A pattern clustering methods from a statistical pattern recognition point of view, with a objective of providing useful advice and references to fundamental ideas available to the broad community of clustering practitioners ${ }^{10}$. The taxonomy of clustering techniques was introduced, and recognizes cross-cutting themes and recent advances. Therefore, the trouble is little prior information about the data, and it is possible to make a few postulations about the data as possible. Clustering is used in several fields to explain process for grouping of unlabeled data. The objective of this concept is to review the core models and methods in the large subset of cluster analysis with its roots in statistics and decision theory.

Unsupervised learning was suggested for distributed clustering ${ }^{11}$. Unsupervised learning is an method of learning, in which the instances are automatically placed into meaningful groups based on their similarity. The intention is to make inferences about its cluster structure, it is necessary to examine whether the data set demonstrates a clustering tendency. Due to the improper measurement, there may be errors in the collected data set. In order to overcome this problem, take an unseen instance, eliminating the value of one of its attributes and then trying to classify it. The missing attribute is calculated to be the same as the value of the attribute on the closest matching instance. This value can be then compared to the actual value of the removed attribute and so can be moderated to be correct or not. This process is repetitive for each attribute. In order to give the average prediction accuracy, the number of attributes properly predicted is then totaled up and divided by the number of attributes.

\section{Document Clustering Approaches}

\subsection{Correlation Preserving Indexing}

Correlation Preserving Indexing is one of the imperative techniques for document clustering. In particular, this method recognizes the manifold structure which is entrenched in the similarities between the set of documents. The semantic constitution is frequently inherent in large number of documents. In the document clustering the most momentous procedure is to determine the intrinsic constitution of the document space. Although the manifold structure is usually entrenched in the similarities between the documents. Correlation is a similarity measure which is significant for confining the manifold structure embedded in the high-dimensional document space. The main purpose of the method is to establish an optimal semantic subspace by concomitantly make use of the associations between the documents in the local patches and to reduce the associations between the documents outside these patches.

The correlation between the two vectors $\mathrm{u}$ and $v$ is as followed as,

$$
\operatorname{Corr}(u, v)=\frac{u^{T} v}{\sqrt{u^{T} u} \sqrt{v^{T} v}}=<\frac{u}{\|u\|}, \frac{v}{\|v\|}>
$$

The correlation corresponds to an angle $\theta$ such that $\operatorname{Cos} \theta=\operatorname{Corr}(\mathrm{u}, \mathrm{v})$. The higher the value of $\operatorname{Corr}(\mathrm{u}, \mathrm{v})$, the stronger the association between the two vectors $\mathrm{u}$ and $\mathrm{v}$. 
1. Online document clustering intend to group documents into clusters, in which the unsupervised learning is converted into semi-supervised learning by using the following information.

A1. In the original document space, if the two documents are close to each other then it grouped into the same cluster.

A2. In the original document space, if the two documents are far away from each other then it grouped into the different clusters.

$y_{i} \in Y$ denotes the low-dimensional illustration of the $\mathrm{i}^{\text {th }}$ document $x_{i} \in X$ in the semantic subspace, in which $\mathrm{i}$ $=1,2, \ldots \mathrm{n}$. Then the above postulation (A1) and (A2) can be described as,

$$
\begin{gathered}
\operatorname{Max} \sum_{i} \sum_{x_{j} \in N\left(x_{i}\right)} \operatorname{Corr}\left(y_{i}, y_{j}\right) \\
\operatorname{Min} \sum_{i} \sum_{x_{j} \in N\left(x_{i}\right)} \operatorname{Corr}\left(y_{i}, y_{j}\right)
\end{gathered}
$$

Correspondingly, where $\mathrm{N}\left(\mathrm{x}_{\mathrm{i}}\right)$ denotes the group of nearest neighbors of $x_{i}$. The optimization of (2) and (3) is analogous to the succeeding metric learning:

$$
d(x, y)=\alpha * \cos (x, y)
$$

In which $\mathrm{d}(\mathrm{x}, \mathrm{y})$ represents the similarity between the documents $\mathrm{x}$ and $\mathrm{y}, \alpha$ denotes the whether $\mathrm{x}$ and $\mathrm{y}$ are the nearest neighbors of each other.

\subsection{Clustering Algorithm based on CPI}

Given the set of documents $x_{1}, x_{2}, \ldots ., x_{n} \in I R^{n}$. The document matrix is represented by $\mathrm{X}$. Based on correlation preserving indexing for document clustering, the algorithm is followed as,

For document clustering based on CPI, the algorithm can be summarizing as follows:

1. Construct the local neighbor patch, and calculate the matrices $M_{S}$ and $M_{T}$ The matrices $M_{S}$ and $M_{T}$ are defined as,

$$
\begin{gathered}
M_{T}=\sum_{i} \sum_{j}\left(x_{i}, x_{j}^{T}\right) \\
M_{S}=\sum_{i} \sum_{x_{j} \in N\left(x_{i}\right)}\left(x_{i}, x_{j}^{T}\right)
\end{gathered}
$$

It is simple to confirm that the matrix $M_{T}$ is semi positive definite. Whereas the documents are expected in the low dimensional semantic subspace in which the correlations between the document points among the nearest neighbors are preserved, this principle can be called as "correlation preserving indexing."

2. The document vectors are assigned into the singular value decomposition subspace by throw away the zero singular values. The representation of the singular value decomposition is as $X=U \sum V^{T}$. All the zero singular values in $\sum$ are eliminated. The vectors in $U$ and $\mathrm{V}$ related to the zero singular values have been eliminated. Therefore the document vectors in the SVD subspace can be accomplished by $\hat{X}=U^{T} X$.

3. Compute CPI Projection. Based on the multipliers $\lambda_{0}, \lambda_{1}, \ldots ., \lambda_{n}$ is obtained. one can compute the matrix $M=\lambda_{0}^{*} M_{T}+\lambda_{1}^{*} x_{1} x_{1}^{T}+\ldots \lambda_{n}^{*} x_{n} x_{n}^{T}$. Let $W_{C P I}$ be the solution of the generalized Eigen value problem $M_{s} W=\lambda M W$. Then, the low dimensional representation of the document can be computed by,

$$
Y=W_{C P I}^{T} \hat{X}=W^{T} X
$$

where, $W=U W_{C P I}$ is the transformation matrix.

4. In the correlation preserving indexing semantic subspace to cluster the documents. While the documents were projected on the unit hyper sphere, the inner product is a natural calculation of similarity. A separation $\left\{\Pi_{j}\right\}_{j=1}^{k}$ of the document can be examined by utilizing the maximization of the subsequent objection function:

$$
Q\left(\left\{\pi_{j}\right\}_{j=1}^{k}\right)=\sum_{j=1}^{k} \sum_{x \in \pi_{j}} x^{T} c_{j}
$$

with $c_{j}=\frac{m_{j}}{\left\|m_{j}\right\|}$ where $m_{j}$ represents the mean of the document vectors enclosed in the cluster $\prod_{j}$.

\subsection{Directed Ridge Regression}

Directed ridge regression is one of the efficient methods that give relationship among the several variables. This method brings the similarity between the documents by measuring the relationship among the variables. The value of regression analysis as a numerical tool may be extensively diminished when the set of independent variables are approximately collinear. Usually in the directed ridge regression method, one can recognize how the characteristic value of the dependent variable modifies when any one of the independent variables is dissimilar, whereas 
the other independent variables are held permanent. The effectiveness of the document clustering is achieved by using the concept of directed ridge regression analysis. In this method it alters only the diagonal elements corresponding to the small Eigen values.

\subsection{Clustering Algorithm based on DRR}

The set of the given documents $x_{1}, x_{2}, \ldots, x_{n I R}{ }^{n}$. Let $\mathrm{X}$ indicates the document matrix. For document clustering based on DRR, the algorithm can be summarizing as follows:

1. Generate the local neighbor patch, and estimate the matrices $M_{S}$ and $M_{T}$.

2. The document vectors are assigned into the singular value decomposition subspace by throw away the zero singular values. The representation of the singular value decomposition is as $X=U \Sigma V^{T}$. All the zero singular values in $\sum$ are eliminated. The vectors in $U$ and $\mathrm{V}$ related to the zero singular values have been eliminated. Therefore the document vectors in the SVD subspace can be accomplished by $\hat{X}=U^{T} X$.

3. After that compute the directed ridge estimator based on the relationship between the Eigen values $U$ and variance $\hat{\alpha}_{i}$. The computation of directed ridge estimator,

$$
\begin{aligned}
\hat{\alpha}(\mathrm{dk})^{(0)} & =(\Lambda+K I)^{-1} \hat{X} \mathrm{U} \\
\mathrm{W} & =\hat{\alpha}(\mathrm{dk})^{*} \hat{X}
\end{aligned}
$$

where, $\mathrm{K}$ is the diagonal matrix.

4. Compute CPI Projection. Based on the multipliers $\lambda_{0}, \lambda_{1}, \ldots ., \lambda_{n}$ is obtained. one can compute the matrix $\mathrm{M}=\lambda_{0}^{*} M_{T}, \lambda_{1}^{*} x_{1} x_{1}^{T}+\ldots .+\lambda_{n}^{*} x_{n} x_{n}{ }^{T}$. Let $W_{C P I}$ be the solution of the generalized eigenvalue problem $M_{S} \mathrm{~W}=$ $\lambda \mathrm{MW}$. Then, the low dimensional representation of the document can be computed by,

$$
\mathrm{Y}=W_{C P I}^{T} \hat{X}=W^{T} \mathrm{X}
$$

where, $\mathrm{W}=U W_{C P I}$ is the transformation matrix.

5. Cluster the documents in the CPI semantic subspace. Since the documents were anticipated on the unit hyper sphere, the inner product is a natural measure of similarity. A partitioning $\left\{\Pi_{j}\right\}_{j=1}^{k}$ of the document is used with the maximization of the following objection function:

$$
Q\left(\left\{\Pi_{j}\right\}_{j=1}^{k}\right)=\sum_{j=1}^{k} \sum_{x \in \Pi j} x^{T} c_{j}
$$

with $c_{j}=c_{j}=\frac{m_{j}}{\left\|m_{j}\right\|}$, where $m_{j}$ is the mean of the document vectors contained in the cluster $\prod_{j}$.

\section{Experimental results}

The Correlation Preserving Indexing and Directed Ridge Regression are compared. The experimental results are attained when the number of nearest neighbors is set to seven or eight. The corresponding results of the correlation preserving indexing and the directed ridge regression are measured for both accuracy and normalized mutual information and the details are shown in Figures 1 and 2.

\subsection{Accuracy}

The accuracy metric (AC) is used to measure the clustering performance. The $\mathrm{AC}$ metric is defined as

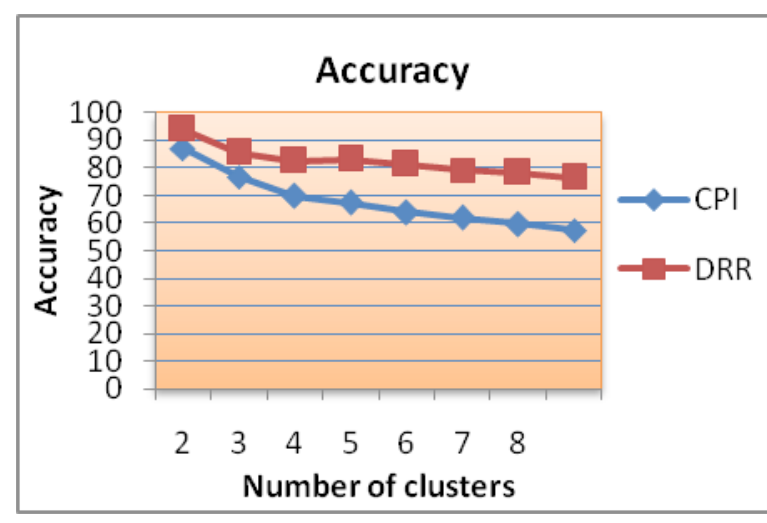

Figure 1. Accuracy comparison.

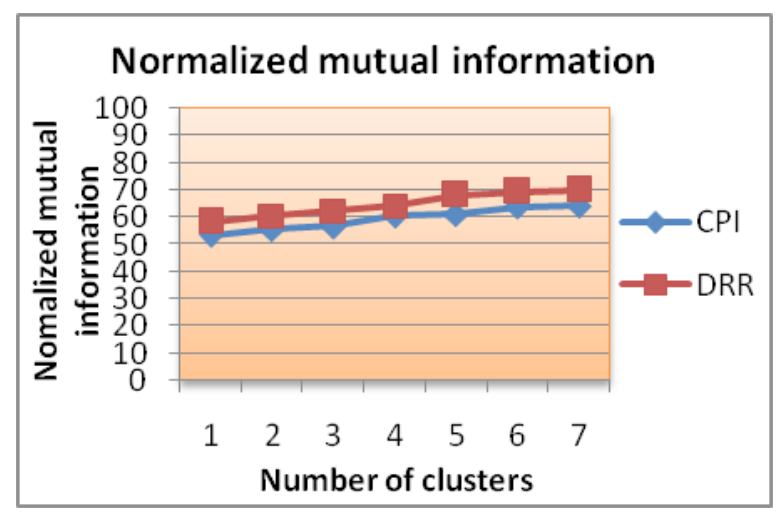

Figure 2. Normalized mutual information. 


$$
\mathrm{AC}=\frac{\sum_{i=1}^{n} \delta\left(S_{i, \text { map }\left(r_{j}\right)}\right)}{n}
$$

where $r_{i}$ is the cluster label attained by the algorithm, $s_{i}$ is label offered by the corpus, $\mathrm{n}$ is the total number of documents, $\delta(x, y)$ is the delta function that equals one if $\mathrm{x}^{1 / 4}$ $\mathrm{y}$ and equals zero otherwise, $\operatorname{map}\left(r_{i}\right)$ is permutation mapping function that maps cluster label $r_{i}$ to the equivalent label from the data corpus.

\subsection{Normalized Mutual Information}

The normalized mutual information $(M I)$

is defined as

$$
\overline{M I}_{\left(\mathrm{C}, \mathrm{C}^{\prime}\right)}=\frac{M I\left(C, C^{\prime}\right)}{\max \left(H(C), H\left(C^{\prime}\right)\right)}
$$

where $\mathrm{C}$ is the set of clusters provided by the document corpus and $\mathrm{C}^{\prime}$ is the set of clusters obtained by the algorithm. $\mathrm{H} \odot$ and $\mathrm{H}\left(\mathrm{C}^{\prime}\right)$ are the entropies of $\mathrm{C}$ and $\mathrm{C}^{\prime}$ respectively. $\mathrm{MI}$ is the mutual information corresponding to the matrices $\mathrm{C}$ and $\mathrm{C}^{\prime}$

$$
\operatorname{MI}\left(\mathrm{C}, \mathrm{C}^{\prime}\right)=\sum_{c i \in C, c_{j}^{\prime} \in C^{\prime}} p\left(C_{i}, C_{j}^{\prime}\right) \cdot \log _{2} \frac{p\left(c_{i}, c_{j}^{\prime}\right)}{p\left(c_{i}\right) \cdot p\left(c_{j}^{\prime}\right)}
$$

Here $\mathrm{p}\left(c_{i}\right)$ is the probability that a document arbitrarily chosen from the corpus belongs to the clusters $c_{i}$ and $\mathrm{p}\left(c_{i}, c_{j}^{\prime}\right)$ is the joint probability that the arbitrarily chosen document belongs to the clusters $c_{i}$ and $c_{j}^{\prime}$ at the same time. It is simple to ensure that $\overline{M I}\left(\mathrm{C}, \mathrm{C}^{\prime}\right)$ takes zero when the two sets are independent, and takes one when the two sets are equal.

\section{Conclusion}

Correlation preserving indexing is one of the innovative approaches for document clustering. In this method the manifold structure is entrenched in the similarities between the documents. Proposed system introduces the similarity between the documents by measuring the relationship among the variables. A directed ridge regression method is one of the effective methods in which capable to predict two or more variables which are highly correlated. An efficient document clustering can be attained by using the directed ridge regression method. Hierarchical fuzzy document clustering can be achieved using a similarity measure of the vectors representing documents.

\section{References}

1. Zhang T, Tang YY, Fang B, Xiang Y. Document clustering in correlation similarity measure space. IEEE Trans Knowl Data Eng. 2012 Jun; 24(6).

2. Liu X, Gong Y, Xu W, Zhu S. Document clustering with cluster refinement and model selection capabilities. Proc $25^{\text {th }}$ Ann Int'l ACM SIGIR Conf Research and Development in Information Retrieval (SIGIR '02); 2002. p. 191-8.

3. Cai D, He X, Han J. Document clustering using locality preserving indexing. IEEE Trans Knowledge and Data Eng. 2005 Dec; 17(12):1624-37.

4. Baker LD, McCallum AK. Distributional clustering of words for text classification. Proc 21st Ann Int'l ACM SIGIR Conf Research and Development in Information Retrieval. 1998. p. 96-103, 1998.

5. Ma Y, Lao S, Takikawa E, Kawade M. Discriminant analysis in correlation similarity measure space. Proc $24^{\text {th }}$ Int'l Conf Machine Learning (ICML '07); 2007. p. 577-84.

6. Zhu X, Ghahramani Z, Lafferty J. Semi-supervised learning using gaussian fields and harmonic functions. Proc 20th Int'l Conf Machine Learning (ICML '03); 2003.

7. Lebanon G. Metric learning for text documents. IEEE Trans Pattern Analysis and Machine Intelligence. 2006 Apr; 28(4):497-507.

8. Xu W, Liu X, Gong Y. Document clustering based on nonnegative matrix factorization. Proc. 26th Ann. Int'l ACM SIGIR Conf Research and Development in Information Retrieval (SIGIR '03); 2003. p. 267-73.

9. Ng RT, Han J. Efficient and effective clustering methods for spatial data mining. Proc 20th Int'l Conf Very Large Data Bases (VLDB); 1994. p. 144-55.

10. Jain AK, Murty MN, Flynn PJ. Data clustering: a review. ACM Computing Surveys. 1999; 31(3):264-323.

11. Kotsiantis S, Pintelas P. Recent advances in clustering: a brief survey. WSEAS Trans Information Science and Applications. 2004; 1(1):73-81. 